\title{
Acrosin release and acrosin activity during incubation in capacitating media using fresh and frozen-thawed dog sperm
}

\author{
Mónica de los Reyes ${ }^{1 *}$, Jaime Palomino ${ }^{1}$, Víctor Martínez ${ }^{2}$, Carolina Aretio ${ }^{1}$, Michel Gutiérrez ${ }^{1}$ \\ 'Laboratory of Animal Reproduction, ${ }^{2}$ Animal Genetics Unit, Faculty of Veterinary Sciences. University of Chile. Santiago, Chile
}

\begin{abstract}
We evaluated the effect of time and temperature on acrosin release from the acrosomal cap and the activity of this enzyme during in vitro capacitation in fresh and frozen/thawed dog sperm. Sperm-rich fractions of six ejaculates from three dogs were processed as fresh and frozen samples. Each sperm sample was incubated in canine capacitation medium (CCM) for $0,1,2$ and $3 \mathrm{~h}$ at $20^{\circ} \mathrm{C}$ and at $37^{\circ} \mathrm{C}$. After incubation, the samples were assessed by the indirect immunofluorescent staining technique. The probability of having unlabeled sperm (PUS), indicating acrosin loss, was modelled by a binomial distribution using logistic regression. There was a linear relationship between PUS and time at both temperatures $(p<0.001)$; however, a major percentage of unlabeled sperm was observed in frozen/thawed samples soon after incubation, indicating that the release of acrosin was affected by capacitation time, mainly in frozen samples. Temperature influenced acrosin release only in cryopreserved sperm $(\mathrm{p}<0.05)$. Acrosin activity was measured by digestion halos on slides coated with gelatin-substrate film during each time period; a significant increase in the number of large halos was observed in fresh samples throughout the experiment, whereas frozen/thawed sperm showed a decreased rate of halo diameters during culture. Thus, there appears to differences between fresh and frozen dog sperm in terms of acrosin release and the level of acrosin activity in the course of in vitro capacitation.
\end{abstract}

Key words: acrosin, capacitation, dog sperm, cryopreservation.

\section{INTRODUCTION}

Before fertilization, mammalian spermatozoa must undergo a process of capacitation, which in vivo occurs in the female reproductive tract. It has also been demonstrated that in dogs, spermatozoa need capacitation as a requisite for fertilization (Mahi and Yanagimachi, 1978; Shimatsu et al., 1992). Capacitation involves many biochemical changes, the principal events associated with this process being: hyperactivated sperm motility and acrosome reaction (Wassarman, 1994; Fraser, 1995). Acrosome reaction is the fusion of the plasma membrane with the outer acrosomal membrane, resulting in vesiculation and the release of acrosomal enzymes that can aid in sperm penetration to the zona pellucida, as well as rendering the spermatozoa capable of fusing with the oocyte plasma membrane (Barros et al., 1992). Zona pellucida glycoproteins induce acrosome reaction in different species (Wassarman, 1994; Moreno et al., 2002) including canines (Kawakami et al., 1993; Brewis et al., 2001), and under in vitro conditions, acrosome reaction may also occur when spermatozoa are incubated in an appropriate medium supporting capacitation (Peña et al., 2004).

Upon acrosome reaction, acrosin, a trypsin-like serine protease present in the acrosome of mature mammalian spermatozoa (Urch, 1991; Gaboriau et al., 2007), is delivered to the extracellular milieu (Harrison et al., 1982; Nuzzo et al., 1990). This enzyme is especially involved in binding and penetration to the zona pellucida during gamete interaction (Barros et al., 1992; De los Reyes and Barros, 2000). In freshly ejaculated sperm, this enzyme is present largely as the inactive precursor, proacrosin, which is converted into acrosin by an intrazymogen mechanism at some time during acrosome reaction (Kennedy and Polakoski, 1981; Urch, 1991).

Many studies have examined changes in acrosin and to some extent proacrosin that occur during or subsequent to the acrosome reaction and capacitation in several species (Harrison et al., 1982; Nuzzo et al., 1990; Valdivia et al., 1994; De los Reyes and Barros, 2000; Moreno et al., 2002; Gaboriau et al., 2007). However, the dog proacrosin/acrosin system has not been extensively studied. Our previous report, using Western blots analysis, suggests that proacrosin is converted to active forms of acrosin earlier in frozen/thawed dog spermatozoa than in fresh spermatozoa during capacitation (De los Reyes et al., 2009). This finding is in accordance with studies that have found cryopreserved dog spermatozoa are capacitated and acrosome reacted at higher rates than fresh spermatozoa during in vitro incubation (Peña et al., 2004). It is possible that the early activation of proacrosin in frozen-thawed sperm induces a premature release of acrosin that reduces fertility.

Although much effort has been directed toward optimizing freezing and thawing techniques in dog semen, attention should also be given to semen processing procedures, considering capacitation protocols, which include time and temperature during culture in order to decrease alterations to the acrosome and plasma membrane, improve post-thaw sperm survival and their ability to fertilize fertilizing. Considering that the acrosin release and activity during incubation in capacitating media may be altered in cryopreserved dog sperm, the objectives of this study were immunolocalize proacrosin/acrosin release and determine the level of acrosin proteolytic activity in fresh and frozen-thawed dog sperm during in vitro capacitation under different culture times and temperatures. 


\section{MATERIAL AND METHODS}

Unless specified, reagents were purchased from Sigma Chemical Co. (St. Louis, MO, USA) and Monoclonal antibody AcrC5F10 was purchased from BIOSONDA (Santiago, Chile). Production of the antibody has been described elsewhere (Valdivia et al., 1994).

This study followed the guidelines for ethical conduct in the care and use of animals instituted by FONDECYT (National Foundation for Sciences and Technology, Chile).

Semen collection and processing

Semen was collected twice using digital stimulation from 3 adult (2-5 years) pure or mix breed dogs. A total of 6 ejaculates were processed separately as one experimental replicate. The sperm rich-fraction of each ejaculate was subjectively determined for progressive motility (using direct observation of a $10 \mu \mathrm{l}$ aliquot of the sample at $200 \mathrm{x}$ ) and morphology using a phase contrast microscope; sperm concentration was measured by a haemocytometer. Only samples with more than $80 \%$ of progressive motility and up to $15 \%$ of abnormal spermatozoa were selected for experiments.

The ejaculate of each dog was processed as a fresh control sample and as a frozen sample. Each sample was diluted 1:2 $(\mathrm{v} / \mathrm{v})$ in Tris buffer medium and centrifuged at $700 \mathrm{X} \mathrm{g}$ for 5 min. The supernatant was discarded and the pellet re-diluted in either canine capacitation medium (CCM) (Mahi and Yanagimachi, 1978) (fresh samples), or in TRIS-citrate fructosefreezing extender (De los Reyes et al., 2006) and processed as frozen samples. Frozen sperm were thawed in water bath at $60^{\circ}$ $\mathrm{C}$ for $8 \mathrm{sec}$, washed by centrifugation at $700 \mathrm{Xg}$ for $5 \mathrm{~min}$ and the pellet of spermatozoa re-suspended in CCM medium, as previously performed in fresh semen. Sperm with post thawing forward motility over $60 \%$ were used for experiments.

Both, fresh and frozen sperm samples were adjusted to a final sperm concentration of 200 million/mL. Each sample (frozen/thawed and fresh) was split into two equal aliquots and incubated for $0,1,2$, and $3 \mathrm{~h}$ at either $20^{\circ} \mathrm{C}$ or $37^{\circ} \mathrm{C}$ separately for sperm capacitation. At each time point, sperm motility was subjectively evaluated with phase contrast microscopy in order to determinate cell viability over time.

\section{Immunofluorescence}

After each culture time in $\mathrm{CCM}$ at $20^{\circ} \mathrm{C}$ or $37^{\circ} \mathrm{C}, 10 \mu \mathrm{L}$ of sperm suspension was assessed for immunofluorescence using human anti-acrosin monoclonal antibody Acr C5F10, which has previously been proven to cross react with canine proacrosin/ acrosin (De los Reyes et al., 2009). In brief, spermatozoa were placed on poly-L-lysine coated slices and after $10 \mathrm{~min}$ fixed in $4 \%$ Paraformaldehyde for $30 \mathrm{~min}$, permeabilized with $100 \%$ methanol for $10 \mathrm{~min}$ and then re-hydrated with $1 \%$ Triton X-100 in PBS for $1 \mathrm{~h}$. Non-specific binding sites were blocked with $1 \%$ bovine serum albumin (BSA) in PBS for 1 $\mathrm{h}$ and after that, incubated with $1 / 1,000$ diluted monoclonal antibody $\mathrm{C} 5 \mathrm{~F} 10$ overnight at $4^{\circ} \mathrm{C}$. The samples were washed in $0.2 \%$ PBS-Tween 20 and incubated for $1 \mathrm{~h}$ at $37^{\mathrm{a}} \mathrm{C}$ in a humid chamber with a second antibody, a goat anti-mouse IgG (dilution 1/100) conjugated with Alexa 488 (Molecular probes, Eugene, OR). The samples were washed thoroughly, and mounted with Vectashield (Vector Laboratories) under coverslips. In each sample, 200 sperm were evaluated under an epifluorescent microscope (UV emission $480 \mathrm{~nm}$ ) (Nikon Optiphot II; Nikon, Kawasaki, Japan).

\section{Acrosin Activity}

At each culture time, acrosin activity was measured by the gelatin-substrate film technique, where enzyme activity is detected by the presence of halos around single sperm resulting from a localized proteolytic digestion of gelatin (Ficsor et al., 1983). As negative controls, sperm samples were incubated under the same conditions for in vitro capacitation with the monoclonal antibody Acr C5F10, dilution 1/ 100.

One milliliter of $2.5 \%$ gelatin suspension was spread on a pre-cooled microscopic slide and fixed for $2 \mathrm{~min}$ in $0.05 \%$ gluteraldehyde, thoroughly washed in PBS, and then kept overnight in a moist chamber at $4^{\circ} \mathrm{C}$. Sperm suspensions $(100$ $\mu \mathrm{l})$ of both fresh and frozen-thawed were placed on the slices and incubated at $38^{\circ} \mathrm{C}$ in $\mathrm{CO}_{2}$ for $24 \mathrm{~h}$. After that, slices were stained with $1 \%$ Comassie Blue for $5 \mathrm{~min}$ and examined under 400X magnification with light microscopy for evidence of digestion. Halo sizes were based on diameter measurements of 100 spermatozoa from each condition and scored with an ocular micrometer (Bausch and Lomb, USA).

\section{Statistical Analysis}

For immunofluorescence technique, 200 sperm were evaluated at each culture time and temperature in fresh and frozenthawed samples, with a total of 16,800 sperm, throughout six experimental replicates. The probability of having unlabeled sperm (PUS) in fresh and frozen-thawed samples was modelled using a binomial distribution with a longitudinal model (dependent on time) using the best fit with logistic regression (Statistical Analysis System, SAS Institute, Cary, NC, USA). For acrosin activity, halo diameter was measured in 100 sperm at each condition. Sperm motility and halo diameters in each type of sperm at each time period were evaluated by ANOVA (Statistical Analysis System, SAS Institute, Cary, NC, USA). The means were compared through Tukey tests. Differences reaching $\mathrm{P}<0.05$ were considered significant.

\section{RESULTS}

\section{Acrosin Release}

Acrosin was localized in the acrosomal cap area when detected by immunostaining using the antibody AcrC5F10. This label appears to be specific because the omission of the first antibody resulted in the absence of fluorescence in the acrosome of the sperm cells (negative control; data not shown).

According to the acrosin immunolabel in the acrosome region of each type of spermatozoa (fresh and frozen-thawed), two sperm patterns were determined as shown in Figure 1: a) fluorescent label over the entire acrosomal region independent of the intensity b) without fluorescent label in the acrosome region. Considering that the sperm were permeabilized, and the antibody recognizes intracellular acrosome-associated acrosin, the first pattern corresponded to spermatozoa that had not completed the acrosome reaction, containing the enzyme inside the acrosome, while those corresponding to the second 
pattern could be spermatozoa that had undergone acrosome reaction and therefore had released acrosin or those dead sperm that lost acrosin as a post-mortem degenerative process.

The results of the logistic analysis of the presence of non-marked sperm related to time (modeled as a linear regression) and the interaction between time and temperature of incubation (for testing heterogeneity of regression slopes), within fresh sperm and frozen-thawed sperm, showed a linear relationship between the probability of having unlabeled sperm (PUS) and time at both temperatures $(\mathrm{p}<0.001)$. This means that from time 0 to $3 \mathrm{~h}$, the expected value of PUS increased under both temperatures (Fig 2A). However, in the frozen-thawed sperm, the effects of the two temperatures were statistically different (Fig $2 \mathrm{~B} ; \mathrm{p}<0.05$ ). The actual mean percentages were in accordance to the predicted probability, because frozen-thawed sperm incubated for $0,1,2$ and $3 \mathrm{~h}$ at the two temperatures $\left(20\right.$ and $\left.37^{\circ} \mathrm{C}\right)$ increased $(\mathrm{p}<0.001)$ the percentage of spermatozoa without fluorescent label from 39.7 $\%$ to $65.4 \%$ and to $73.4 \%$ at $20^{\circ} \mathrm{C}$ and at $37^{\circ} \mathrm{C}$ respectively. Thus, both time of culture and temperature significantly influenced each capacitation procedure. In fresh sperm, as the incubation time increased, the percentage of unlabeled spermatozoa also increased $(\mathrm{p}<0.001)$ from $13.9 \%$ at time 0 , to $35.7 \%$ and $32.1 \%$ at the $3 \mathrm{~h}$ at $20^{\circ} \mathrm{C}$ and $37^{\circ} \mathrm{C}$ respectively,

1

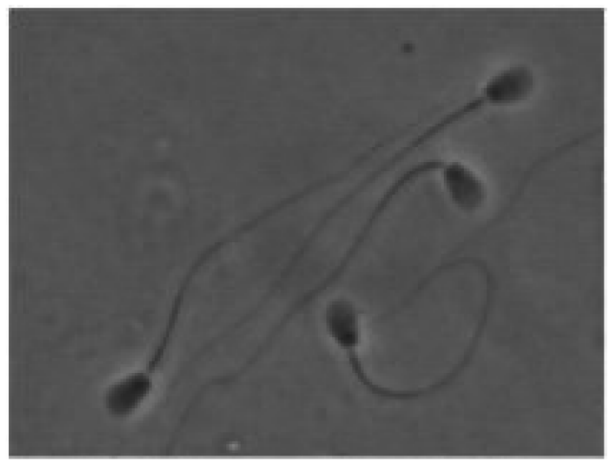

2

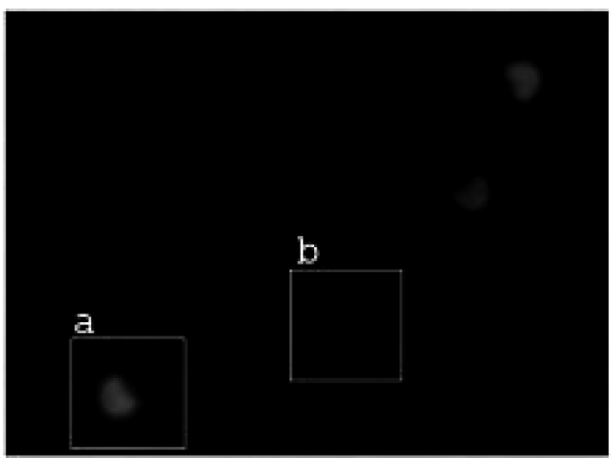

Figure 1: Indirect immunofluorescent staining of dog sperm during in vitro capacitation using human anti-proacrosin/acrosin monoclonal antibody AcrC5F10 and observed by (1) phase contrast and (2) epifluorescence microscopy where one can see labeled (a) and unlabeled sperm (b). Magnification X 1000. without differences between the two incubation temperatures $(p>0.05)$. Differences $(p<0.05)$ in the percentages of unlabeled sperm were observed during all culture periods between frozen-thawed and fresh samples.

\section{Sperm Motility}

In each capacitation procedure (considering time and temperature) sperm viability was subjectively determined through sperm motility. In frozen samples, decreased motility was observed when incubation time and temperature increased $\left(67.1 \%\right.$ to $41.4 \%$ and to $25.7 \%$ at $20^{\circ} \mathrm{C}$ and $37^{\circ} \mathrm{C}$ from beginning to the third hour, respectively; $\mathrm{p}<0.05)$. In fresh samples, progressive motility also decreased over time (81\% to $50 \%$ at $20^{\circ} \mathrm{C}$ and $81 \%$ to $53 \%$ at $\left.37^{\circ} \mathrm{C} ; \mathrm{p}<0.05\right)$, but the incubation temperature did not have a significant effect $(\mathrm{p}>0.05)$.

\section{Acrosin Activity}

Fresh and frozen-thawed dog spermatozoa incubated at the two temperatures for up to $3 \mathrm{~h}$, displayed digestion halos on

A

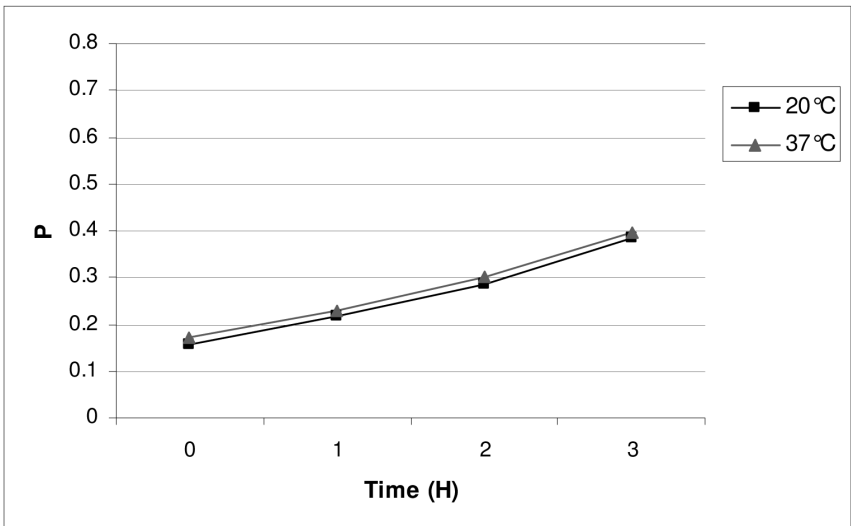

B

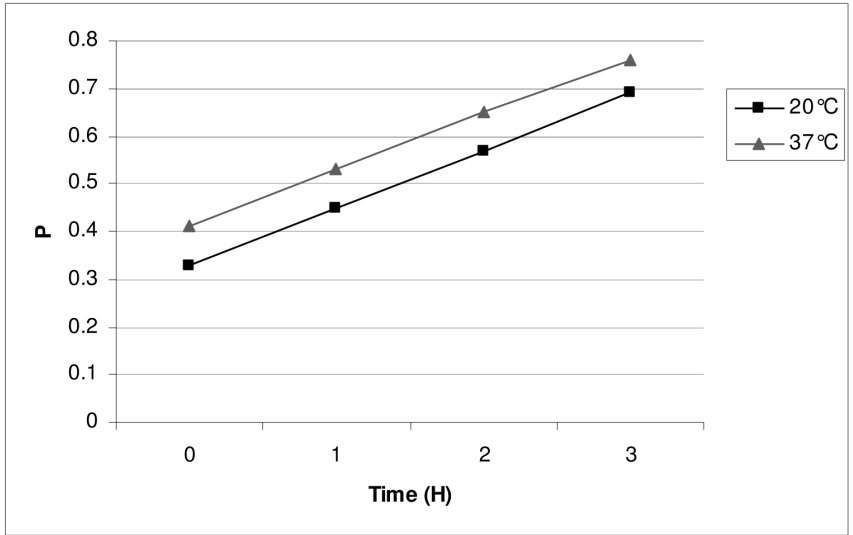

Figure 2. The predicted binomial probability $(P ; Y$ axis) of unlabeled sperm expressed as a function of time ( $X$ axis). A) Fresh Sperm; both lines were not statistically different $(p>0.05)$. B) frozen/thawed sperm; both lines were statistically different $(p<$ 0.05). There was a significant time trend in both analyses. 
gelatin film. The localized digestion of the gelatin was due to acrosin since the antibody Acr C5F10 against acrosin inhibited such digestion in control samples. The digestion halos showed different sizes (Fig. 3). Acrosin activity as measured by halo diameter on slides coated with gelatin showed a significant difference between fresh and frozen sperm (Fig 4; $\mathrm{p}<0.05$ ). The mean halo diameter was smaller in fresh samples in comparison to frozen-thawed samples at the beginning of culture (time 0 and 1). During the following culture times (2 and $3 \mathrm{~h}$ ), an increase in halo diameter was observed in fresh sperm, being wider than those observed in frozen-thawed sperm, which in contrast, showed a decrease in mean halo size. Incubation temperature did not influence either fresh or frozen-thawed sperm.

\section{DISCUSSION}

Capacitation prepares the sperm to undergo acrosome reaction with the accompanying activation and release of lytic enzymes exposing membrane receptors (De los Reyes and Barros, 2000; Moreno et al., 2002). In this study, the effect of time and temperature on acrosin release and activity during in vitro capacitation of fresh and frozen-thawed dog sperm was evaluated. Monoclonal antibody AcrC5F10 was used to immunolocalize the proacrosin/acrosin system in spermatozoa prior to and during capacitation. Acrosin immunofluorescent staining was distributed over the acrosomal cap in agreement with other works (Barros et al., 1992; Cortes et al., 2006). The degree to which anti-acrosin antibody cross-reacted with zymogen and with the different active forms of acrosin is relevant, because the degree to which the enzyme can be detected depends on this. In a previous study, we showed that the antibody used here reacted strongly with dog proacrosin, as well as its active forms (De los Reyes et al., 2009). Thus, the major forms of acrosin were detected by immunofluorescence technique under capacitation conditions. Proteolytic activity of acrosin was also evaluated under the same conditions by gelatin substrate assay, which depends on the functional properties of the acrosomal membrane, as well as the state and amount of enzyme within the acrosome (Ficsor et al., 1983; Welker et al., 1988).

We found that the release of acrosin was highly associated with the extent to which dog spermatozoa were incubated. In both fresh and frozen-thawed sperm, the fluorescence stain

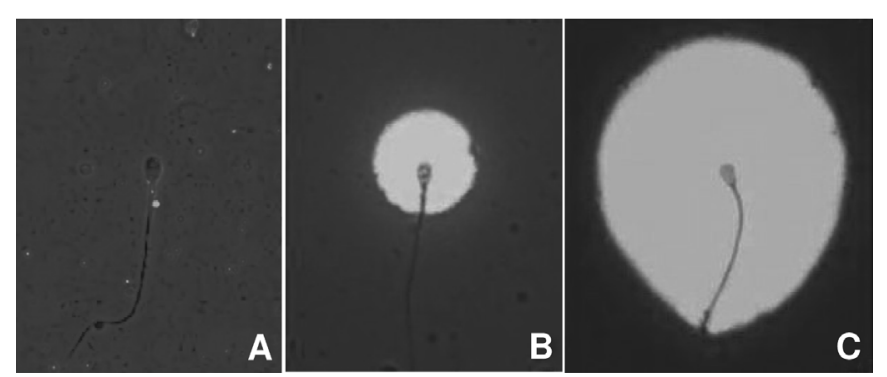

Figure 3: Photomicrograph (magnification: $400 \mathrm{x}$ ) of canine spermatozoa after incubation on gelatin substrate slides $A$, spermatozoa showing good acrosin activity, large halo diameter; $B$, spermatozoa showing regular acrosin activity, medium and small halo diameter ; C, spermatozoa showing no halo formation. decreased with time, corresponding to sperm that had lost acrosin.

Previous works have described that the release of inactive proacrosin from the acrosome matrix appears associated with the conversion of this protein to the enzymatically active acrosin protease (Nuzzo et al., 1990; Urch, 1991). Our recent report using Western immunoblots analysis in dog semen, provided evidence that beta-acrosin reactivity in fresh sperm was directly proportional to the capacitation time. However, in frozen sperm the reactivity of beta-acrosin was strongest at the beginning of culture, suggesting that proacrosin is converts to the active form of the enzyme at a higher rate in frozen-thawed than in fresh spermatozoa (De los Reyes et al., 2009). The differences found between frozen and fresh dog spermatozoa in the present study are in agreement with our previous findings. This is because, although the percentage of unlabeled spermatozoa increases with time in both fresh and frozen spermatozoa, acrosin was rapidly lost from the acrosome in cryopreserved samples soon after incubation. However, in fresh samples acrosin remained associated with the sperm for longer periods as the majority of sperm heads with fluorescent stain was largely present at the beginning of culture.

We cannot discount, however, the possibility that some of the non-labeled frozen-thawed sperm represent degenerated or dead cells resulting in the loss of acrosin due to reduced membrane permeability and not as a consequence of capacitation. Nevertheless, the percentage of post-thaw motility was near $70 \%$ and at this time point spermatozoa without immunofluorescent label were almost $40 \%$. Therefore, there was a proportion of living frozen-thawed sperm that released acrosin soon after thawing, possibly as a result of increased susceptibility to stimuli, inducing the acrosomal reaction in which all the acrosome reacted and the acrosin was dissolved or eliminated from the acrosomal region.

It has been described that cryopreservation induces capacitation-like changes (Watson, 2000; Sirivaidyapong et al., 2000; Peña et al., 2004). More capacitated and acrosomalreacted dog spermatozoa are present earlier when sperm is cryopreserved than in fresh sperm during in vitro incubation (Rota et al., 1999; Peña et al., 2004), although it is still unclear whether spermatozoa are actually capacitated or merely

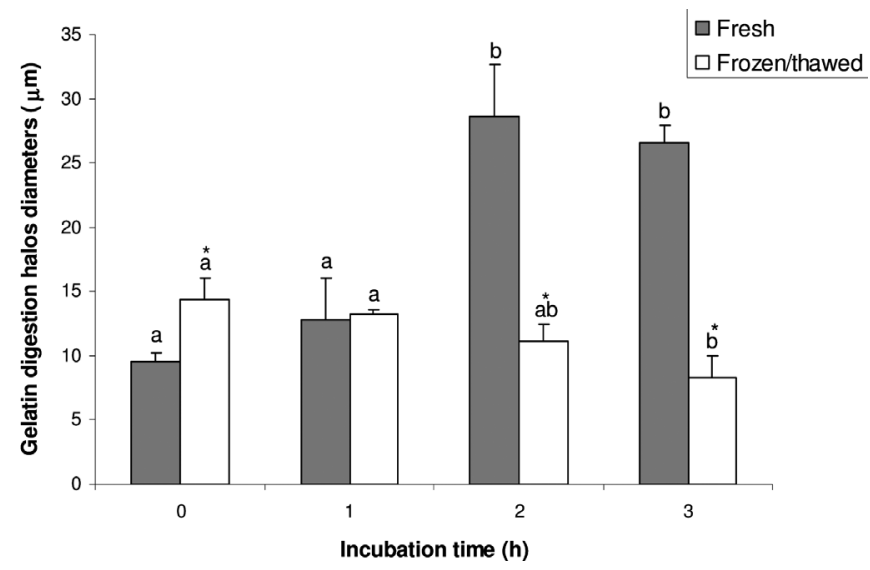

Figure 4: Mean of halo diameter \pm SD of proteolytic activity on gelatin films using fresh and frozen/thawed dog spermatozoa during in vitro capacitation. 
proceed directly to acrosomal exocytosis. In the present study, using frozen-thawed sperm, the release of acrosin was higher throughout cultures when compared to fresh sperm; but acrosin activity, assessed by the presence of halo around single sperm resulting from localized proteolytic digestion of gelatin, was greater than among frozen-thawed sperm than among fresh sperm only at the beginning of culture, which is consistent with previous studies that demonstrated the strongest reactivity of beta acrosin occurs soon after thawing in dog semen (De los Reyes et al., 2009). Hence, it is reasonable to assume that only such active acrosin can be involved in this sperm function. Thus, even though a significant proportion of the proacrosin/acrosin may be lost or destroyed by freezingthawing, the remaining activity is still higher than that of fresh spermatozoa at the beginning of culture. However, fresh spermatozoa retained their acrosin activity over time, since the presence and the sizes of gelatin halos increased throughout incubation periods. This increasing gelatin digestion observed in fresh samples agrees well with the results of Kawakami et al., (1999), who concluded that acrosin activity in fresh dog sperm, measured with light spectrophotometry, increased in association with sperm capacitation.

Apparently, activation of proacrosin to acrosin does not occur within the intact acrosome, but rather is initiated after physical disruption of the acrosome. The higher rate of unlabeled spermatozoa in frozen-thawed sperm in comparison to fresh spermatozoa during all culture periods is more likely due to an acrosome alteration induced by freezingthawing procedures, as has been described previously in non-capacitated dog spermatozoa (Florman et al., 1984; Cortes et al., 2006). Acrosin is considered a diffusible molecule (Harrison et al., 1982), and the release of acrosin into seminal plasma during the aging of human semen has been reported (Schill and Fritz, 1975), indicating that sperm with some degree of disruption are more likely to release acrosin. Mechanisms regulating hydrolase release from spermatozoa are poorly understood at the molecular level, however it is known that freeze-thaw procedures damage the acrosome and spermatozoa membranes, inducing the influx of calcium into the cell (Peña et al., 2004), due in part to decrease of calcium ATPase activity (Cormier et al., 1997). Calcium exerts a stimulatory effect on acrosin activity in boar (Kennedy and Polakoski, 1981) and bovine (Nagdas, 1992; Rosatti et al., 2003) sperm, so that more proacrosin/acrosin is exposed to substrates. Therefore, total proacrosin/acrosin release would be useful primarily as an indicator of acrosomal alteration and is probably less useful as a fertility parameter than acrosin activity, determined in the present study by means of this gelatinolytic technique, because it is conceivable that proacrosin conversion to acrosin cannot leave the total acrosin content intact, but rather greatly decreases the amount of active acrosin available to the spermatozoa (Goodpasture et al., 1981).

Capacitation is described as a temperature-dependent process, in some species like human, sperm incubation at room temperature does not allow capacitation. However, this blocking effect is overcome when spermatozoa are exposed to $37^{\circ} \mathrm{C}$ (Maryn-Briggiler et al., 2002). In contrast, storage of ram semen at room temperature has a marked capacitating effect on a subpopulation of spermatozoa (Perez et al., 1997). The most common temperature used for capacitation in in vitro protocols has been $37-38^{\circ} \mathrm{C}$. In this study, sperm samples were incubated at $37^{\circ}$ and $20^{\circ} \mathrm{C}$. At both temperatures, fresh and frozen- thawed sperm showed the same trend in the percentage of immunolabeling over time. However, temperature significantly influenced the rate of acrosin release in cryopreserved sperm. In this population, incubation at $37^{\circ} \mathrm{C}$ enhanced acrosin release since a higher percentage of unlabeled sperm was observed at $37^{\circ}$ than at $20^{\circ} \mathrm{C}$ during all culture periods. Sirivaidyapong et al., (2000) also found that acrosome reaction in cryopreserved dog sperm occurred more rapidly at $37^{\circ} \mathrm{C}$ than at $20^{\circ} \mathrm{C}$. Energy metabolism is influenced by temperature increasing glucose consumption and glucose has been reported to favor acrosome reaction in dog spermatozoa (Fraser, 1995).

However, acrosomal proteolytic activity determined by means of the gelatinolytic technique was not influenced by preincubation temperature in either fresh or frozen dog sperm. In the same way, it has been reported that no significant changes in acrosin activity are observed when human spermatozoa are maintained at 3 to $6^{\circ} \mathrm{C}$ or at 22 to $24^{\circ} \mathrm{C}$ over a 24 -hour period. A six-hour storage at $37^{\circ} \mathrm{C}$ did not alter the acrosin activity either (Kennedy et al., 1989). The present results show that dog ejaculates can be preincubated at either $20^{\circ} \mathrm{C}$ or $37^{\circ} \mathrm{C}$ for capacitation without significant change in the acrosin activity. However, the observed effect on acrosin release in frozen-thawed sperm when incubation temperature is raised at $37^{\circ} \mathrm{C}$, is probably sustained by major susceptibility to changes induced during capacitation, reflected also in reduced cell viability over time, owing to alterations of the plasma and acrosomal membranes. Freezing-thawing acts mainly by disordering the lipid structure of the membranes and loss of cholesterol from the sperm plasma membrane, which has a destabilizing effect on sperm and may be part of the molecular mechanism of capacitation in vivo (Iborra et al., 2000). Removal of cholesterol from bovine sperm increases sensitivity to a lysophosphatidylcholine challenge and induction of the acrosome exocytosis (Foote and Parks, 1993).

Since sperm capacitation, and thus acrosome reaction, is required for fertilization, premature release and activity of acrosin may impair fertilization, because they cannot retain their fertilizing ability over time (Barros et al., 1992; Palomino and De los Reyes, 2009).

In summary, our results provide support that there is a difference between fresh and frozen dog sperm in terms of the release of acrosin and the level of acrosin activity of acrosin over the course of in vitro capacitation. Premature activation and early and high release of acrosin could be other functional parameters affected by cryopreservation.

\section{ACKNOWLEDGMENTS}

This work was supported by grants FONDECYT 1060602 and 1080618 to MDLR.

\section{REFERENCES}

BARROS C, CAPOTE C, PÉREZ C, CROSBY J, BECKER MI, DE IOANNES A (1992) Immunodetection of acrosin during the acrosome reaction of hamster, guinea pig and human spermatozoa. Biol Res 25: 31-40.

BREWIS IA, MORTON IE, MOORE HD, ENGLAND GC (2001) Solubilized zona pellucida proteins and progesterone induce calcium influx and the acrosome reaction in capacitated dog spermatozoa. Mol Reprod Dev 60: 491-497.

CORMIER N, SIRARD MA, BAILEY J (1997) Premature capacitation of bovine spermatozoa is initiated by cryopreservation. J Androl 18: 461467. 
CORTÉS CJ, CODELIA VA, MANOSALVA I, DE LANGE J, DE LOS REYES M, MORENO RD (2006) Proacrosin/acrosin quantification as an indicator of acrosomal integrity in fresh and frozen dog spermatozoa. Anim Reprod Sci 93:165-175.

DE LOS REYES M, BARROS C (2000) Immunolocalization of proacrosin/ acrosin in bovine sperm and sperm penetration through the zona pellucida. Anim Reprod Sci 58: 215-228.

DE LOS REYES M, CARRION R, BARROS C (2006) In vitro fertilization of in vitro matured canine oocytes using frozen-thawed dog semen. Theriogenology 66: 1682-1684.

DE LOS REYES M, MEDINA G, PALOMINO J (2009) Western blot analysis of proacrosin/acrosin in frozen dog sperm during in vitro capacitation. Reprod Domest Anim 44 (Suppl 2): 278-283.

FICSOR G, GINSBERG LC, OLDFORD BS, SNOKE RE, BECKER RW (1983) Gelatin-Substrate film technique for detection of acrosin in single mammalian sperm. Fertil Steril 39: 548-552.

FOOTE RH, PARKS JE (1993) Factors affecting preservation and fertility of bull sperm: a brief review. Reprod Fertil Develop 5: 665-673.

FRASER LR (1995) Cellular biology of capacitation and the acrosome reaction. Hum Reprod 10 (Suppl 1): 22-30.

FROMAN DP, AMANN RP, RIEK PM, OLAR TT (1984) Acrosin activity of canine spermatozoa as an index of cellular damage. J Reprod Fertil 70: 301-308.

GABORIAU D, HOWES EA, CLARK J, JONES J (2007) Binding of sperm proacrosin/ $\beta$-acrosin to zona pellucida glycoproteins is sulfate and stereodependent. Synthesis of a novel fertilization inhibitor. Dev Biol 306: 646-657.

GOODPASTURE JC, REDDY JM, ZANEVELD LJ (1981) Acrosin, proacrosin and acrosin inhibitor of guinea pig spermatozoa capacitated and acrosome reacted in vitro. Biol Reprod 25: 44-45.

HARRISON RAP, FLECHON JE, BROWN CR (1982) The location of acrosin and proacrosin in ram spermatozoa. J Reprod Fertil 66: 349-358.

IBORRA A, COMPANYO M, MARTÍNEZ P, MORROS A (2000) Cholesterol efflux promotes acrosome reaction in goat spermatozoa. Biol Reprod 62: 378-383.

KAWAKAMI E, ARAI T, OISHI I (1999) Changes in Hyaluronidase, Acrosin and N-Acetylhexosaminidase activities of dog sperm after incubation. J Vet Med Sci 61: 183-184.

KAWAKAMI E, VANDEVOOT CA, MAHI CA, OVERSTREET JW (1993) Induction of acrosome reaction of canine sperm by homologous zona pellucida. Biol Reprod 48: 841-845.

KENNEDY WP, KAMINSKI, VAN DERVEN HH, JEYENDRAN RS, REID DS, BLACKWELL JM, BIELFELD PJ, ZANEVELD LJD (1989) A simple clinical assay to evaluate the acrosin activity of human spermatozoa. J Androl 10: 221-230.

KENNEDY WP, POLAKOSKI KL (1981) Evidence for an intrazymogen mechanism in the conversion of proacrosin into acrosin. Biochemical 20: 2240-2245.

MAHI CA, YANAGIMACHI R (1978) Capacitation, acrosome reaction, and egg penetration by canine spermatozoa in a simple defined medium. Gamete Res 1: 101-109.
MARYN-BRIGGILER CI，TEZON JG，MIRANDA PV，VÁZQUEZ-LEVIN $\mathrm{MH}$ (2002) Effect of incubating human sperm at room temperature on capacitation-related events. Fertil Steril 77: 252-259.

MORENO RD, BUSTAMANTE E, SCHATTEN G, BARROS C (2002) Inhibition of mouse in vitro fertilization by an antibody against a unique 18-amino acid domain in the polysulfate-binding domain of proacrosin/ acrosin. Fertil Steril 77: 812-817.

NAGDAS SK (1992) Bovine epididymal sperm proacrosin-acrosin system: quantification and partial characterization. Andrologia 24: 111-121.

NUZZO NA, ANDERSON RA, ZANEVELD LJ (1990) Proacrosin activation and acrosin release during the guinea pig acrosome reaction. Mol Reprod Dev 25: 52-60.

PALOMINO J, DE LOS REYES M (2009) A scanning electron microscopy study of frozen/thawed dog sperm during in vitro gamete interaction. Reprod Domest Anim 44: 278-283.

PEÑA AI, BARRIO M, BECERRA JJ, QUINTELA LA, HERRADÓN PG (2004) Zona pellucida binding ability and responsiveness to ionophore challenge of cryopreserved dog spermatozoa after different periods of capacitation. Anim Reprod Sci 84: 193-210.

PÉREZ LJ, VALCÁRCEL A, DE LAS HERAS MA, MOSES D, BALDASSARRE $H$ (1997) The storage of pure ram semen at room temperature results in capacitation of a subpopulation of spermatozoa. Theriogenology 47: 549-558.

ROSATTI MI, BECONI MT, CÓRDOBA M (2003) Proacrosin-acrosin activity in capacitated and acrosome reacted sperm from cryopreserved bovine semen. Biocell 28: 311-316.

ROTA A, PEÑA AI, LINDE-FORSBERG C, RODRÍGUEZ-MARTÍNEZ H (1999) In vitro capacitation of fresh, chilled and frozen-thawed dog spermatozoa assessed by the chlortetracycline assay and changes in motility patterns. Anim Reprod Sci 57: 199-215.

SCHILL WB, FRITZ H (1975) N-benzoyl-L-arginine ethil ester splitting activity (acrosin) in human spermatozoa and seminal plasma during aging in vitro. Hoppe Seyler's Z Physiological Chemical 356, 83-90.

SHIMATSU Y, YAMADA S, KAWANO Y, NAKAZAMA M, MAITO K, TOYODA Y (1992) In vitro capacitation of canine spermatozoa. J Reprod Develop 38: 67-71.

SIRIVAIDYAPONG S, CHENG FP, MARKS A, VOORHOUT WF, BEVERS M, COLENBRANDER B (2000) Effect of sperm diluents on the acrosome reaction in canine sperm. Theriogenology 53: 789-802.

URCH UA (1991) Biochemistry and function of acrosin. In: The Biology and Chemistry of Mammalian Fertilization. Ed. P.M Wassarman pp 233-248. Chicago, CRC Press.

VALDIVIA M, YUNES R, MELÉNDEZ J, DE IOANNES A, LEYTON L, BECKER MI, BARROS C (1994) Immunolocalization of proacrosin/ acrosin in rabbit sperm during acrosome reaction and in spermatozoa recovered from the perivitelline space. Mol Reprod Dev 37: 216-222.

WASSARMAN PM (1994) Gamete interactions during mammalian fertilization. Theriogenology 41: 31-44.

WATSON PF (2000) The causes of reduced fertility with cryopreserved semen. Anim Reprod Sci 60-61: 481-492.

WELKER B, BERNSTEIN GS, DIEDRICH K, NAKAMURA RM, KREBS D (1988) Acrosomal proteinase activity of human spermatozoa and relation of results of semen quality. Hum Reprod 3 (Suppl 2): 75-80. 